\title{
Vehicle Identification using Fuzzy Adaline Neural Network
}

\author{
${ }^{1}$ Gokul Murugesan, ${ }^{2}$ Shriram K. Vasudevan, ${ }^{3}$ Sivaraman Ramachandran, \\ ${ }^{4}$ Subashri Vasudevan and ${ }^{5}$ Balachandran Arumugam \\ ${ }^{1}$ MTech, CVIP, Amrita University, Coimbatore, India \\ ${ }^{2}$ Department of Computer Science, Amrita University, Coimbatore, India \\ ${ }^{3}$ Technical Faculty, Corporate and Industry Relations, Amrita University, Bangalore, India \\ ${ }^{4}$ M.Tech, MNC, Chennai, India \\ ${ }^{5} \mathrm{CIR}$, Amrita University,Coimbatore, India
}

Received 2013-04-23, Revised 2013-05-06; Accepted 2013-06-06

\begin{abstract}
Video surveillance is an important aspect in today's world, where a particular scene of area requires monitoring to avoid terrorist attacks and unauthorized entries. Vehicle recognition is an important area in object tracking and recognition. Objects may be of rigid or non-rigid in nature with varying velocity and have different features. Important features like shape, logo, color and texture are complex in nature. Hence there is a need of better algorithm for detecting and identifying the objects like car. A new method is proposed for recognizing the cars present in the video. At first the features like shape is extracted using moments, logo using the Scale Invariant Feature Transform (SIFT) and the RGB color values of the car body. Using these features the recognition is carried out to classify the type of car. Recognition of cars has range of application like, military surveillance, traffic management, autonomous navigation system, auto parking.
\end{abstract}

Keywords: SIFT, Feature Descriptors, ADALINE, Fuzzy

\section{INTRODUCTION}

In today's world surveillance has become an important work of interest specially, when it comes to monitoring vehicles only the manual observation of the videos are used or an automated system of number plate recognition (Zhanga and Wangb, 2012; Zhu et al., 2012; Parisi et al., 1998; Porikli and Kocak, 2006) are currently being used. What if the entire system can be automated? This gave the authors a motivation for developing a system where, when a car arrives its shape, color and make information is extracted and a description is given. The proposed approach extracts three features the shape using moments, logo using SIFT feature extraction and color using the RGB value ranges.

This study is organized as:
- Feature extraction

- Moments

- Proposed architecture

- Results

\subsection{Feature Extraction}

Features are the basic identity of an image; they almost describe an image uniquely. The features should carry enough information about the image and should not require any domain-specific knowledge for their extraction. They should be easy to compute in order for the approach to be feasible for a large image collection and rapid retrieval. There are various feature extraction techniques like, Scale Invariant Feature Transform (SIFT). Speeded up Robust

Corresponding Author: Gokul Murugesan, MTech, CVIP, Amrita University, Coimbatore, India 
Features (SURF), Harris Corner, Canny Edge Detection. The SIFT feature extraction was used for our problem. SIFT features are invariant to scale rotation and also view point variations hence even the camera angle varies by a small amount the feature extraction would be feasible and accuracy would be large.

\subsection{Moments}

Moments can also be used as a feature extraction technique. Moment is a certain particular weighted average of the image pixels' intensities, usually chosen to have some attractive property or interpretation. An essential issue in the field of pattern analysis is the recognition of objects and characters Regardless of their position, size and orientation. The idea of using Moments in shape recognition gained prominence when derived a set of invariants using algebraic invariants. Two-dimensional moments of a digitally sampled $M \times M$ image that has gray function $\mathrm{f}(\mathrm{x}, \mathrm{y}),(\mathrm{x}, \mathrm{y}=0, \ldots \mathrm{M}-1)$ is given as:

$$
\mathrm{m}_{\mathrm{pq}}=\sum_{\mathrm{x}=0}^{\mathrm{x}=\mathrm{M}-1} \sum_{\mathrm{y}=0}^{\mathrm{y}=\mathrm{M}-1}(\mathrm{x})^{\mathrm{p}} \cdot(\mathrm{y})^{\mathrm{q}} \mathrm{f}(\mathrm{x}, \mathrm{y}) \mathrm{p}, \mathrm{q}=0,1,2,3 \ldots
$$

The moments $f(x, y)$ translated by an amount $(a, b)$, are defined as:

$$
\mu_{p q}=\sum_{x} \sum_{y}(x+a)^{p} \cdot(y+b)^{q} f(x, y)
$$

Thus the central momentsm $\mathrm{pq}_{\mathrm{pq}}$ or $\mu_{\mathrm{pq}}$ can be computed from the above equation on substituting $\mathrm{a}=-\mathrm{x}$ and $b=-y$ as:

$$
\overline{\mathrm{x}}=\frac{\mathrm{m}_{10}}{\mathrm{~m}_{00}} \text { and } \overline{\mathrm{y}}=\frac{\mathrm{m}_{01}}{\mathrm{~m}_{00}}, \mu_{\mathrm{pq}}=\sum_{\mathrm{x}} \sum_{\mathrm{y}}(\mathrm{x}-\overline{\mathrm{x}})^{\mathrm{p}} \cdot(\mathrm{y}-\overline{\mathrm{y}})^{\mathrm{q}} \mathrm{f}(\mathrm{x}, \mathrm{y})
$$

When a scaling normalization is applied the central moments change as:

$$
\eta_{\mathrm{pq}}=\mu_{\mathrm{pq}} / \mu_{\mathrm{o0}}^{\gamma}, \gamma=[(\mathrm{p}+\mathrm{q}) / 2]+1
$$

\subsection{Proposed Architecture}

\subsubsection{Algorithm}

Figure 1 Describes the architecture for the proposed system:
Step 1: Video containing cars is given as input

Step 2: The video is split into frames for further processing

Step 3: Each frame is sent for feature extraction

Step 4: Feature extraction

Feature extraction is of three stages:

Stage1: The Shape is extracted using the contour detection and the detected contour of the car is used to identify the moment value of the shape. This moment value is fed as input to the fuzzy based ADALINE neural network. Figure 5 describes the result of shape extraction for Suzuki swift car.

\section{Algorithm for Shape Extraction:}

Step 1: Each frame is first converted to a binary image using an adaptive Thresholding where the colour image is converted into a black and white image.

Step 2: The binary image is used to identify the contour of the car using the cv Find Contour function

Step 3: The identified contour is applied Hu-moments to get the feature value Fig. 4 describes the $\mathrm{Hu}$ moments values for Suzuki Swift car as input

Step 4: The error in the feature values (i.e., the amount that it does not belong to a particular shape) and the change in error is given as input to the Fuzzy Adaline neural network, based on the membership function and the error rate the neural network identifies it as to which model it belongs.

Stage 2: The Logo is extracted from the original colour frame using the SIFT feature descriptors. The descriptor's error and change in error is given as the input to the fuzzy ADALINE neural network. These descriptors gives us which make the care belongs to)

\subsection{Algorithm for Logo Identification}

Step 1: The extracted frame is taken as input to the SIFT feature extraction. Figure 2 shows the feature calculation for the three brands used

Step 2: The logo area is found out by using the trained xml file.

Step 3: The identified logo is done a SIFT based matching with the templates found. 
Gokul Murugesan et al. / Journal of Computer Science 9 (6): 757-762, 2013

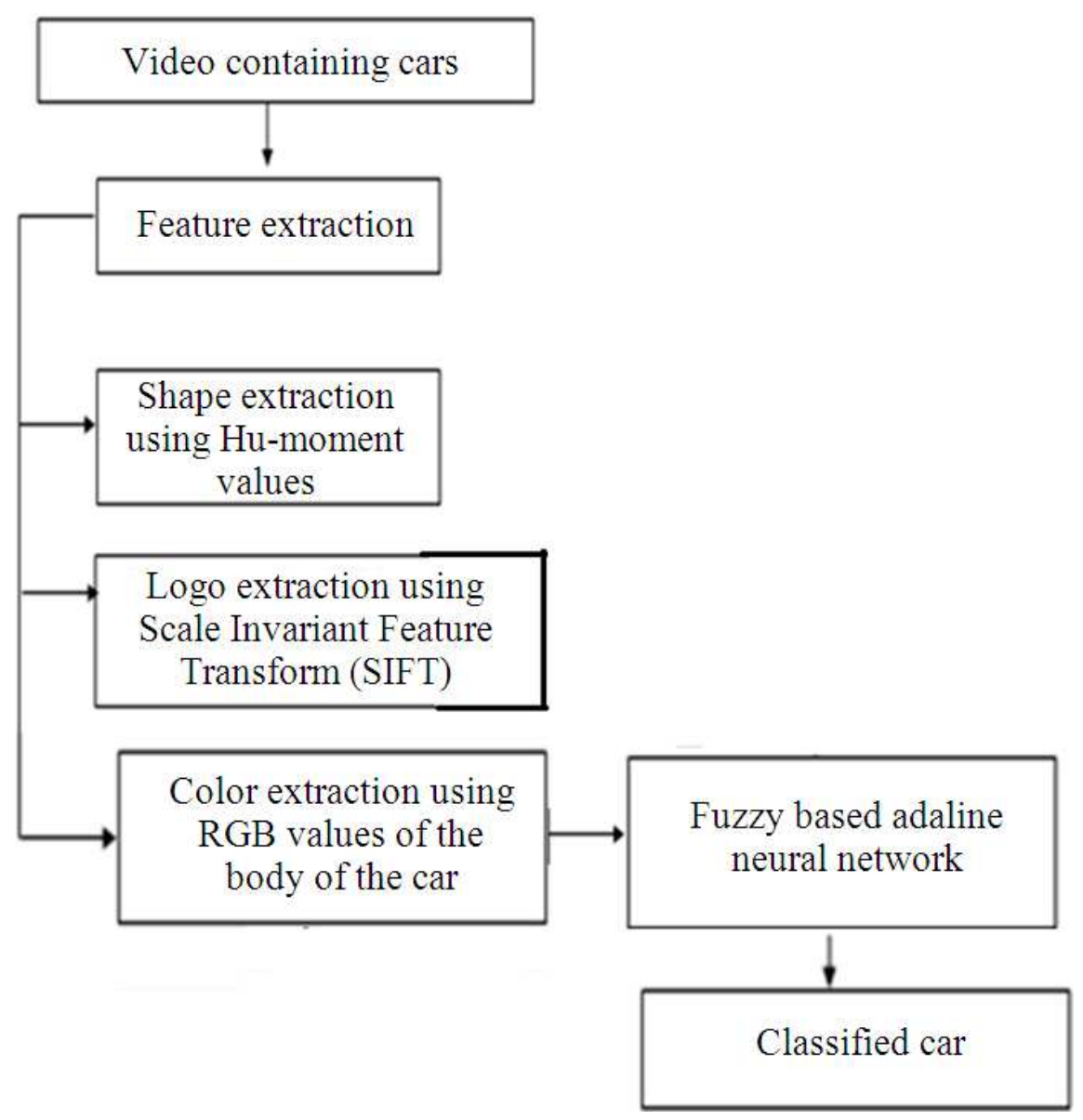

Fig. 1. Proposed architecture flow diagram

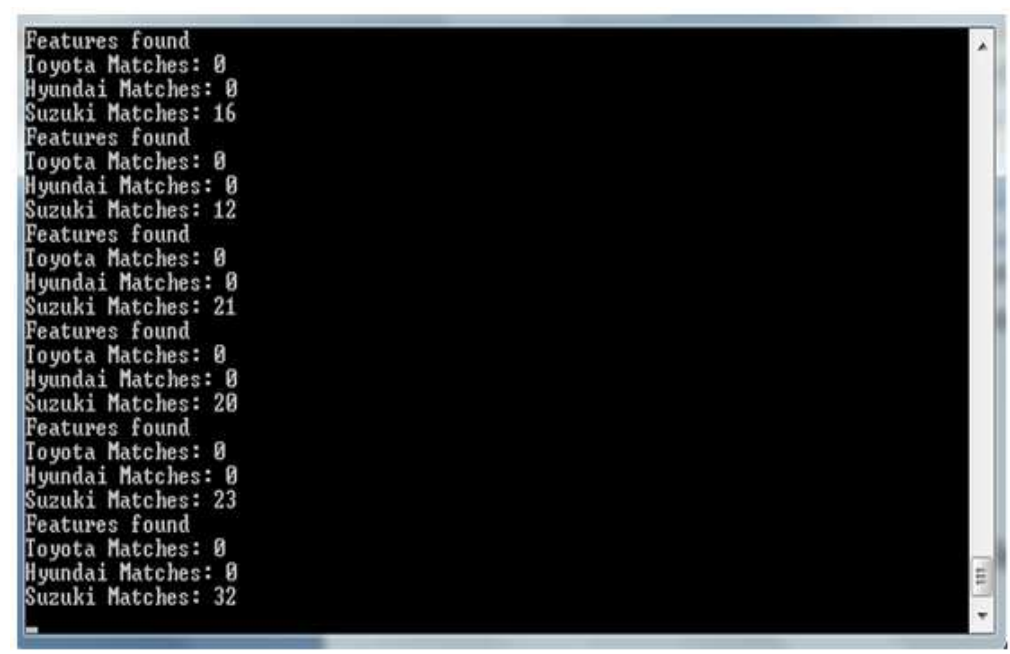

Fig. 2. Feature calculation for the logo templates 


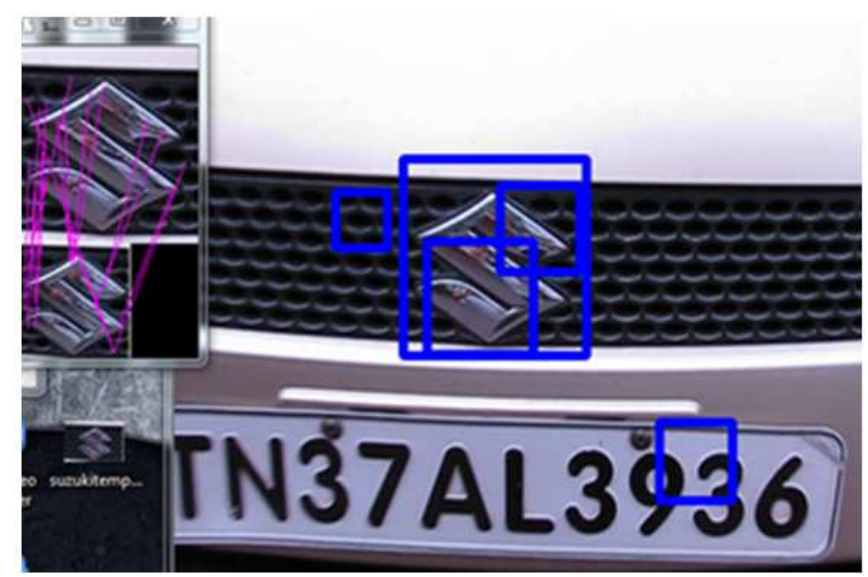

Fig. 3. The detected feature of the Suzuki logo

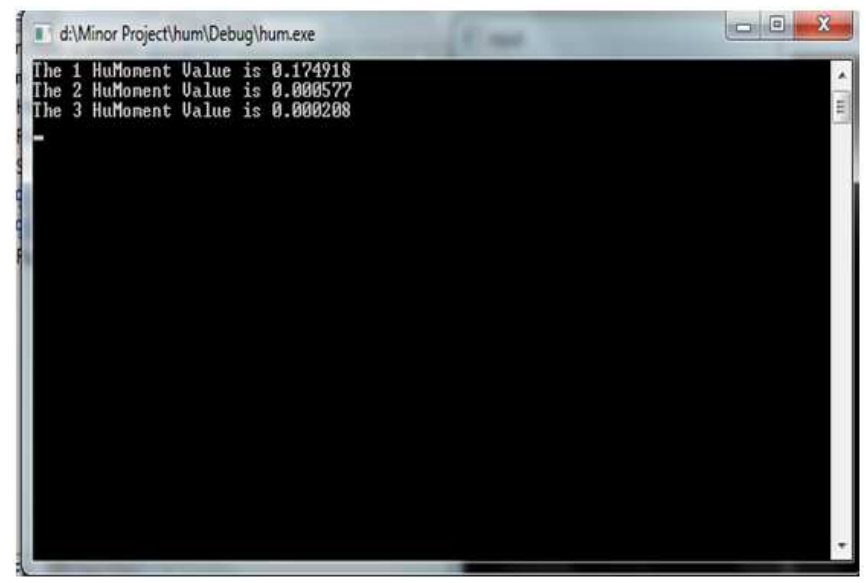

Fig. 4. The detected Hu moments value

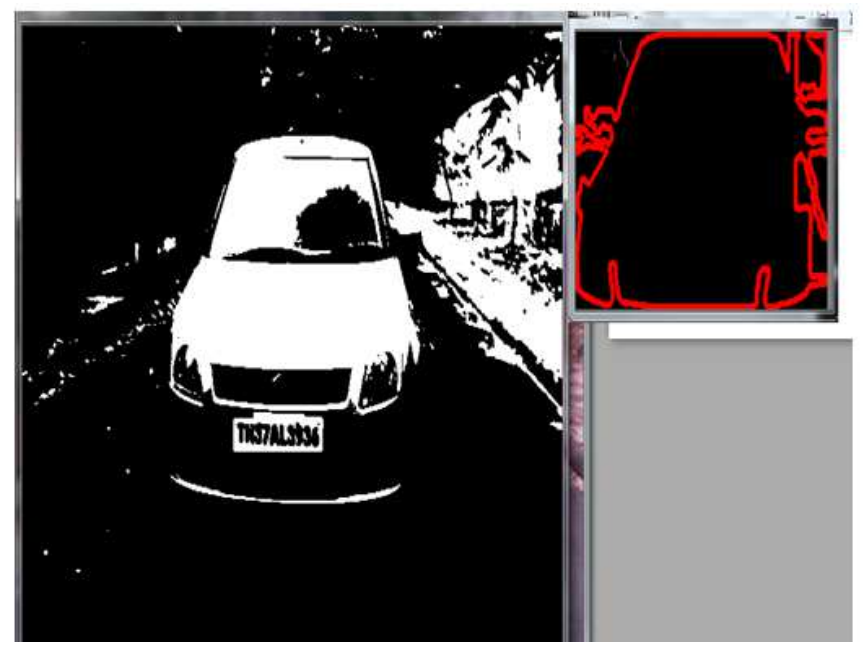

Fig. 5. The detected feature of the Shape for one frame 
Table 1. Tabulation of results obtained

\begin{tabular}{lllllll}
\hline Parameter & $\begin{array}{l}\text { Suzuki } \\
\text { logo }\end{array}$ & $\begin{array}{l}\text { Hyundai } \\
\text { shape }\end{array}$ & $\begin{array}{l}\text { Toyota } \\
\text { logo }\end{array}$ & Shape & Logo & Shape \\
\hline $\begin{array}{l}\text { Total no of detected } \\
\text { features }\end{array}$ & 134 & 143 & 166 & 106 & 154.0 & 123.0 \\
$\begin{array}{l}\text { Total no of relevant } \\
\text { features obtained }\end{array}$ & 122 & 48 & 147 & 61 & 135.0 & 56.0 \\
$\begin{array}{l}\text { Precision } \\
\text { Recall }\end{array}$ & $91 \%$ & $33 \%$ & $88 \%$ & $57 \%$ & $87.6 \%$ & $45.5 \%$ \\
\hline
\end{tabular}

Step4: The amount of match for each make (brand) of the car is found by the SIFT matching. Figure 3 shows the logo matching of.

Step5: The error or misclassification and the change in error of the match is taken as the input to the fuzzy ADALINE (Xindong et al., 2008; Psyllos et al., 2010; Jeatrakul and Wong, 2009) neural network.

Stage 3: The colour of the car is taken by counting the pixel values of the contour region of the car. The pixel value that has the largest count is the colour of the car.

\subsection{Algorithm for Colour Feature Extraction}

Step 1: The input frame and the contour frame identified in the stage 1 is given as input for colour identification.

Step 2: The area of the contour is traced in the colour frame and the pixel values are counted

Step3: The bin which has the maximum number of counts is considered as the identified colour.

Step4: The identified colour and the change in the error values as shown below is considered as the input to the ADALINE neural network. e.g., say the maximum color bin is white i.e., $255,255,255$

White ratio $=$ the amount of white pixels/the total number of pixels in contour area. Error is the other pixel values present in the area i.e., (1-white ratio). This error is considered as the input to the ADALINE neural network. The output of these stages is considered as the input to the fuzzy ADALINE neural network (Xindong et al., 2008; Jeatrakul and Wong, 2009; Sinthupinyo et al., 2009).

Step5: The feature error values are given as the input to the fuzzy based ADALINE neural network

The parameter of the neural network are as follows. Learning rule:

$$
\mathrm{w}(\mathrm{k}+1)=2 \times \mathrm{a} \times \mathrm{e}(\mathrm{k}) \mathrm{p}(\mathrm{k}) \quad \mathrm{b}(\mathrm{k}+1)=\mathrm{b}(\mathrm{k})+2 \times \mathrm{a} \times \mathrm{e}(\mathrm{k})
$$

Output:

$$
y=\sum_{i=1}^{4} x(i) w(i)=x^{T} w
$$

\section{Error Function}

$$
\mathrm{e}=\mathrm{r}-\mathrm{y}=\mathrm{r}-\mathrm{x}^{\mathrm{T}} \mathrm{w}
$$

Weight function:

$$
w(t+1)=w(t)+\text { Fuzzy }(e, d e)
$$

Where:

$\mathrm{x}(\mathrm{i})=$ Input variable

$\mathrm{w}(\mathrm{t})=$ Weight obtained at time instance $\mathrm{t}$

$\mathrm{b}(\mathrm{t})=$ Weight bias value at time instance $\mathrm{t}$

$\mathrm{w}(\mathrm{t}+1)=$ Weight at the next time instance $\mathrm{t}+1$

$\mathrm{b}(\mathrm{t}+1)=$ Weight bias value at the time instance $\mathrm{t}+1$

e $\quad=$ Error variable

de $\quad=$ Difference in error

Fuzzy (e,de)-fuzzy set operation between the error and the change in errory-output value.

Step 6: The output of the fuzzy ADALINE neural network (Paul et al., 1998) classifies the output as to which brand it belongs to the color of the car and the model of the car.

\section{RESULTS}

The developed system was tested with the videos obtained using a standard video camera in a controlled environment and the results are as below.

\section{CONCLUSION}

Car were detected and recognized based on the shape, color and logo features that are extracted from the frames 
of the input video. This method can detect and recognize Hyundai i10, Suzuki Swift and Toyota Innova. The Output will be the brand, color of the car and the model of the car are detected. Table 1 discusses about the results obtained after classification for three brand of cars. In future the scope of this work, various brands needs to be tested and speeding of the feature extraction procedure will be carried out.

\section{REFERENCES}

Jeatrakul, P. and K.W. Wong, 2009. Comparing the performance of different neural networks for binary classification problems. Preceedings of the 8th International Symposium on Natural Language Processing, Oct. 20-22, IEEE Xplore Press, Bangkok, pp: 111-115. DOI: 10.1109/SNLP.2009.5340935

Parisi, R.E.D., D. Claudio, G. Lucarelli and G. Orlandi, 1998. Car plate recognition by neural networks and image processing. Proceedings of the IEEE International Symposium on Circuits and Systems, May 31-Jun 3, IEEE Xplore Press, Monterey, pp: 195-198. DOI: 10.1109/ISCAS.1998.703970

Paul, B., A. Konar and A.K. Mandal, 1998. Image recognition with fuzzy adaline neurons. Proceedings of the IEEE World Congress on Computational Intelligence Neural Networks Proceedings, May 4-8, IEEE Xplore Press, pp: 747-752. DOI: 10.1109/IJCNN.1998.682374
Porikli, F. and T. Kocak, 2006. Robust license plate detection using covariance descriptor in a neural network framework. Proceedings of the IEEE International Conference on Video and Signal Based Surveillance, (BS' 06), IEEE Xplore Prees, pp: 107107. DOI: 10.1109/AVSS.2006.100

Psyllos, A.P. and C.N.E. Anagnostopoulos, 2010. Vehicle logo recognition using a sift-based enhanced matching scheme. Proceedings of the IEEE Transactions on Intelligent Transportation Systems, (TS' 10), IEEE Xplore Press, pp: 322-328. DOI: 10.1109/TITS.2010.2042714

Sinthupinyo, S., W. Roadrungwasinkul and C. Chantan, 2009. User recognition via keystroke latencies using SOM and backpropagation neural network sukree. Proceedings of the International Joint Conference, Aug. 18-21, IEEE Xplore Press, ICROS-SICE, Fukuoka, pp: 3160-3165.

Xindong, W., V. Kumar, J.R. Quinlan, J. Ghosh and Q. Yang et al., 2008. Top 10 algorithms in data mining.

Zhanga, Z. and C. Wangb, 2012. The research of vehicle plate recognition technical based on BP neural network. AASRI Proc., 1: 74-81. DOI: 10.1016/j.aasri.2012.06.013

Zhu, Y., H. Huang, Z. Xu, Y. He and S. Liu, 2012. Chinese-style plate recognition based on artificial neural network and statistics advanced in control engineering and information science. Proc. Eng., 15: 3556-3561. 